\title{
Striking a new path
}

\author{
Gerhard Rechkemmer
}

Published online: 2 August 2011

(C) Springer-Verlag 2011

Since the European Journal of Nutrition (EJN) was launched more than 12 years ago, it has constantly developed and is well established among the major peer-reviewed journals in nutrition and dietetics. Thanks also to the excellent work of our editorial board and the editorial office, the impact factor of EJN has continually increased over the period. The recently announced 'impact factor 2010 ' has jumped to 3.343 , and EJN is now listed as number 16 among 70 journals in nutrition and dietetics. This is a great achievement.

The focus of EJN has been and still is on human nutrition from basic, mechanistic studies, experimental data from model organisms to dietary intervention and epidemiological studies in humans.

However, as in other fields, on the one hand there is a growing diversification and the need for researchers to concentrate on specific fields of expertise, while on the other hand researchers still need to oversee the major developments and findings in the whole field of nutrition. To adapt to this trend and hence make EJN even more attractive to contributors and readers and to further stimulate the submission of excellent manuscripts, EJN recently revised its editorial policy by assigning section editors for specific scientific subject matters. Also, some new members have joined the editorial board. The new sections and the responsible section editors are:

- Biomarkers and DNA damage (Andrew Collins)

- Carcinogenesis (Piero Dolara)

G. Rechkemmer ( $\square)$

Max Rubner-Institut, Federal Research Institute of Nutrition and Food, Haid-und-Neu-Str. 9, 76131 Karlsruhe, Germany e-mail: ejn@mri.bund.de
- Molecular physiology and cell signalling (Bernard Corfe)

- Immunology and inflammation (Dirk Haller)

- Lipid metabolism (Klaus Eder)

- Metabolomics; Micronutrients (Marc Ferrara)

- Nutrigenomics (Michael Müller)

- Probiotics and gut health (Ian Rowland)

- Regulation of food intake (Thomas Lutz)

- Sports and nutrition (Peter Stehle)

This change implies that the individual editors will assume a greater responsibility for the further development of their respective sections. With the new competences, we not only hope to further increase the scientific merit of EJN but also improve the review process, leading to timely decisions about acceptance or rejection of submitted manuscripts. One advantage of this new concept of EJN is that it can be dynamically adjusted to the most recent developments in nutritional sciences. New sections and responsible editors will be quickly added in the future if important new topics in nutrition research evolve. Furthermore, editors are encouraged to invite topical reviews for their specific sections, so that our readers can benefit from a growing number of in-depth review papers on hot topics from various fields. This new concept has already been put into practice in the past four issues of EJN in 2011. The published articles and reviews have already resulted in a significant increase in online downloads, indicating the importance of the new concept for the international recognition of EJN.

With the joint efforts of the editorial and the international advisory boards, the editorial assistant (Silvia Roser) and the editorial office (Daniela Oberreuther-Moschner), we are aiming to further increase the international reputation of EJN. I encourage scientists active in human 
nutrition research to submit their original work and review papers to EJN and we will ensure a competent and quick review and, after acceptance, rapid publication.
Gerhard Rechkemmer

Editor-in-Chief 